\title{
„Und für Opa sorgt 'ne Frau aus Osteuropa.“ Die stille Globalisierung der Familienarbeit
}

\author{
ELISABETH BECK-GERNSHEIM
}

\section{Globalisierung der Hausarbeit: Wie kommen Angebot und Nachfrage zustande?}

Nach den Verwüstungen des Zweiten Weltkriegs erlebten viele westliche Länder in den 1950er und 1960er Jahren eine Phase großen wirtschaftlichen Aufschwungs. Für die expandierende Wirtschaft wurden dringend Arbeitskräfte gesucht - und wo man sie im eigenen Land nicht mehr fand, holte man sie von anderswo her. In der Folge verließen viele Menschen aus den wirtschaftlich schwächeren Ländern ihre Heimat, um in den stärker industrialisierten Ländern Arbeit und bessere Lebenschancen zu finden. Die meisten übernahmen ungelernte oder angelernte Tätigkeiten in der Industrie; und die meisten von ihnen waren Männer.

Seit einiger Zeit erleben wir nun eine neue Form der Arbeitsmigration - beginnend ungefähr in den 80er Jahren. Da sind Frauen aus Mexiko, die in Kalifornien als Kindermädchen arbeiten; Philippinas, die in Israel alte Menschen betreuen; Frauen aus Polen, die in deutschen Haushalten putzen, waschen und bügeln - die Arbeit, um die es hier geht, findet vorwiegend in privaten Haushalten statt. Nicht wenige von denen, die diese Arbeit verrichten, können eine qualifizierte Ausbildung aufweisen; aber aufgrund der wirtschaftlichen Bedingungen in ihrer Heimat haben sie dort kaum Aussicht auf ein gesichertes Einkommen. 


\section{Wohlstandsgefälle und politische Umbrüche}

Hinter dieser neuen Form der Arbeitsmigration stehen verschiedene Ursachen. Die erste liegt auf der Hand. Es ist dieselbe, die auch die frühere Welle der Arbeitsmigration in Gang setzte, nämlich das Wohlstandsgefälle zwischen den reicheren und den ärmeren Ländern. Doch anders als in den 1950er und 1960er Jahren werden heute in den hochindustrialisierten Ländern kaum noch ungelernte oder angelernte Arbeitskräfte gesucht. Im Gegenteil, viele Industriezweige sind von Wirtschaftskrisen und Umstrukturierung betroffen; immer mehr Betriebe üben sich in so genannten „Rationalisierungsmaßnahmen“. In der Folge sind viele Arbeitsplätze verschwunden, und zwar gerade auch in solchen Bereichen, wo vorher Migranten eingestellt wurden.

Hinzu kommen die Veränderungen auf der politischen Landkarte Europas. Mit dem Zusammenbruch des Sozialismus sind in den ehemals sozialistischen Staaten viele Arbeitsplätze entfallen, die vorher staatlich subventioniert waren, aber unter den neuen Bedingungen nicht mehr finanziert werden können. Ob in Russland, in der Ukraine, in Polen immer mehr Menschen in Osteuropa sind nun von Arbeitslosigkeit betroffen. Immer mehr Menschen dort wissen nicht, wie sie sich und ihre Familie ernähren sollen.

Doch der Ausweg, der sich in früheren Jahrzehnten bot - der Weg in die Industrien des Westens - dieser Ausweg ist heute versperrt. So wird nun ein anderer gesucht, eben der in die privaten Haushalte des Westens.

\section{Die Arbeitsteilung im Privaten}

Das führt zum zweiten Ursachenbündel, zur Arbeitsteilung zwischen Männern und Frauen. In den 1950er und 1960er Jahren war die so genannte traditionelle Arbeitsteilung die Norm: der Mann der Ernährer, die Frau zuständig vor allem für Heim und Familie. Als dann die neue Frauenbewegung aufkam, setzte sie ein anderes Leitbild dagegen. Die vorherrschende Arbeitsteilung wurde einer umfassenden Revision unterzogen und grundsätzlich in Frage gestellt. Beide Geschlechter, so die neue Vision, sollten an beiden Bereichen teilhaben. Und das hieß im Klartext: Männer sollten die Arbeit im Privaten mit übernehmen. Sie sollten putzen, waschen, kochen und Kinder wickeln.

Seit damals hat sich zweifellos manches verändert. Aber ebenso zweifellos ist, dass der Wandel - wenn man von wenigen Ausnahmen absieht - sehr begrenzt blieb.

Wie einschlägige Studien zeigen, haben viele Männer der jüngeren Generation tatsächlich ein engeres Verhältnis zu ihren Kindern entwi- 
ckelt. Sie spielen mehr mit ihnen, sie bringen sie morgens zum Kindergarten oder abends zu Bett. Aber dieselben Studien zeigen, dass es bis heute vorwiegend Frauen sind, die den größten Teil der Kinderversorgung und -erziehung übernehmen. Und dies gilt umso mehr für die allgemeinen Aufgaben im Haushalt; da erst recht bleibt die Beteiligung der Männer bescheiden - und dies auch dann, wenn die Frauen berufstätig sind. Das Ergebnis ist, um den Begriff der amerikanischen Soziologin Arlie Hochschild zu verwenden, eine „unfertige soziale Revolution“ (Hochschild/Machung 1990: 34) im Bereich der Geschlechterbeziehungen; oder auch eine "Schieflage in der Geschlechtszuständigkeit“ (BMFSFJ 2005: 175), wie es im Siebten Familienbericht der Bundesregierung heißt.

\section{Notlagen und Überlebensstrategien}

Weil aber die berufstätigen Frauen nicht alles allein leisten können, suchen sie die Unterstützung anderswo. Und das heißt: von anderen Frauen. Die neue Arbeitsteilung im Privaten, die sich in den letzten Jahrzehnten etabliert hat, sieht so aus: Die Frauen der Mittelschicht, gut ausgebildet und berufsmotiviert, delegieren einen Teil der Familienaufgaben an Hilfskräfte. Um den Alltag zu bewältigen, werden oft ganze Netzwerke von Unterstützerinnen eingesetzt (Tagesmutter, AupairMädchen, Babysitter, dazu Schwester und Schwiegermutter als letzte Reserve). Und zumindest in den Städten gilt, die Helferinnen kommen immer seltener aus dem direkten Umfeld, sondern oft von weit her. Es sind Frauen aus der Zweiten und Dritten Welt, die in der Ersten Welt Erwerbschancen suchen: Frauen aus Polen oder Rumänien, aus Mexiko oder Sri Lanka, die in Hongkong, Rom oder New York Arbeiten im privaten Haushalt verrichten - eine Wanderungsbewegung von den armen zu den reichen Nationen.

Diese Entwicklung ist, so die Soziologin Maria S. Rerrich, das Ergebnis eines ,patriarchalen Webfehlers“ (Rerrich 1993: 333) in den politischen und institutionellen Vorgaben unserer Gesellschaft. Zum einen haben wir familienfeindliche Strukturen auf dem Arbeitsmarkt, darüber hinaus sind Bildungs- und Sozialpolitik so aufgebaut, dass sie wesentlich auf die unentgeltliche private Dienstbereitschaft von Frauen setzen. Das schafft erhebliche Belastungen im Alltag Millionen berufstätiger Frauen. Diese werden deshalb - sofern sie es sich irgendwie leisten können - notgedrungen jeweils individuelle Entlastung suchen. Auf der anderen Seite existiert eine Ausländerpolitik, die vielen Migrantinnen keine anderen Erwerbschancen als die in privaten Haushalten ermöglicht. Beides zusammengenommen schafft eine Nachfragesituation, in der 
häusliche Arbeit zwischen verschiedenen Gruppen von Frauen neu aufgeteilt wird und in der Männer überwiegend außen vor bleiben.

\section{Alternde Gesellschaft}

Hinzukommt, dass in den letzen Jahrzehnten die Lebenserwartung kontinuierlich gestiegen ist. Da mit höherem Alter die physischen Kräfte nachlassen, Krankheiten und chronische Beschwerden zunehmen, wächst gleichzeitig die Zahl der Menschen, die für ihre alltägliche Versorgung auf Unterstützung durch andere angewiesen sind - sei's gelegentlich und stundenweise, sei's täglich und rund um die Uhr. Aber die Plätze in Seniorenheimen und Pflegeheimen sind teuer; und die Versorgung ist dort oft unzulänglich.

In dieser Situation bieten die ausländischen Helferinnen einen willkommenen Ausweg: Statt Massenabfertigung lockt persönliche Betreuung rund um die Uhr und zu einem bezahlbaren Preis. So verwundert es nicht, dass gerade der Pflegebereich zu einem Arbeitsmarkt für Migrantinnen geworden ist - Tendenz: schnell steigend.

\section{Quantitativer Umfang}

Eine solche Angabe lädt zum Nachfragen ein: Wie groß ist der entsprechende Markt? Wie viele Hausarbeitsmigrantinnen gibt es eigentlich?

Die erste Antwort heißt: Genaue Zahlen haben wir nicht. Die zweite Antwort heißt: Wir haben es sicherlich nicht mit einer kleinen Gruppe zu tun. Nach einschlägigen Schätzungen beschäftigen etwa 7,6\% der Privathaushalte in Deutschland regelmäßig eine Putz- oder Haushaltshilfe, weitere $4 \%$ tun dies hin und wieder. Zusammengenommen entspricht dies mehr als vier Millionen Beschäftigtenverhältnissen. Obwohl diese einen erheblichen Teil des Arbeitsmarktes ausmachen, liegen dazu bisher erst wenige Untersuchungen vor. Jedoch gehen die Experten übereinstimmend davon aus, dass ein großer Teil der Beschäftigten Migrantinnen sind.

\section{Eine Win-win-Situation?}

Man kann die bisher beschriebene Geschichte positiv deuten, als Wohltat für alle. Dank der wachsenden Zahl von Arbeitsmigrantinnen können Frauen der Ersten Welt einen Teil der Aufgaben von Haushalt und Kindererziehung delegieren. Gleichzeitig können Frauen aus ärmeren Ländern mit dem Geld, das sie hierzulande verdienen, für sich und ihre Fa- 
milien die Basis für eine bessere Zukunft schaffen. So profitieren beide Seiten, oder schön neudeutsch gesagt: eine Win-win-Situation.

Eine solche Sichtweise ist angenehm, weil sie uns - die Bewohner und Bewohnerinnen der Ersten Welt - von unbequemen Fragen befreit. Die Frage ist nur, ob diese harmonische Deutung auch stimmt. Dazu will ich nun die Umstände und Folgen genauer betrachten.

\section{In den Grauzonen der Legalität: die Situation der Migrantinnen im Aufnahmeland}

Da sich die westlichen Nationen durch immer restriktivere Migrationsgesetze nach außen abzuschotten versuchen, bewegen sich viele Migrantinnen in den Grauzonen zwischen Legalität und Illegalität. Entsprechend prekär und unsicher ist ihr Status, vielfach von Entdeckung und Ausweisung bedroht. Weil sie keine Alternativen auf dem offiziellen Arbeitsmarkt haben, müssen sie für vergleichsweise geringe Entlohnung arbeiten. Um dennoch möglichst viel zu verdienen, müssen sie umso länger und mehr arbeiten. Darüber hinaus können sie leicht zum Opfer von Ausbeutung werden und sind kaum in der Lage, sich dagegen zu wehren - weil sie mit den einschlägigen Gesetzen nur spärlich vertraut sind; weil sie oft nur geringe Sprachkenntnisse haben; und weil sie jeden Kontakt mit offiziellen Institutionen vermeiden, um ja nicht die Abschiebung zu riskieren.

Der Migrationsexperte Klaus J. Bade hat diesen Zusammenhang auf drei Worte zusammengefasst: „Fleißig, billig, illegal“ (Bade 2000) - so beschreibt er die Stellung illegaler MigrantInnen.

\section{Bewusste Duldung und Pakt des Schweigens}

Und genau deswegen - weil sie viel leisten und wenig kosten - werden Migrantinnen beschäftigt. Davon profitieren durchaus nicht nur die Frauen der Ersten Welt mit ihren Berufswünschen, sondern mindestens ebenso die dazugehörigen Männer. Sie erst recht können beruflichen Ambitionen nachgehen, unbelastet von störenden Fragen, von der Forderung nach Beteiligung beim Waschen-Putzen-Pflegen. Es ist eine Art Pakt des Schweigens, ein wechselseitiges Stillhalte-Abkommen der Geschlechter: Wenn die Frauen der Ersten Welt es schaffen, dass die private Alltagsarbeit einigermaßen funktioniert, dann „dürfen“ sie beruflich engagiert sein und sogar höhere berufliche Ziele verfolgen. Und umgekehrt: Wenn die dazugehörigen Männer ihren beruflichen Ambitionen 
nicht im Weg stehen, dann organisieren die Frauen die private Alltagsarbeit, und zwar durch Delegation nach außen, statt das Binnenverhältnis der Paarbeziehung mit unbequemen Forderungen zu belasten.

Machen wir dazu ein Gedankenexperiment: Wenn es die Migrantinnen aus Polen oder Rumänien, aus Mexiko oder Honduras nicht mehr gäbe; wenn sie allesamt das täten, was der Gesetzgeber des Ankunftslandes von ihnen erwartet; wenn sie morgen in ihre Heimat zurückkehren würden - was wäre dann? Dann würde es wohl nicht mehr reichen, dass die deutschen oder die US-amerikanischen Männer verbal die Norm der Gleichberechtigung hochhalten. Stattdessen wären diese Männer weit mehr auch mit den alltagspraktischen Folgen dieser Norm konfrontiert. Dann steht weit drängender die Frage im Raum: Wann machst du was? Wann putzt du das Bad, wann kümmerst du dich einmal um Vater, wann begleitest du das Kind zur Krankengymnastik? In diesem Sinne kann man sagen: Indem die Migrantinnen die unsichtbare Hintergrundarbeit in der Familie übernehmen, stabilisieren sie den prekären Frieden im Geschlechterverhältnis.

In ähnlichem Sinne profitieren auch der Staat bzw. die Politiker, Kommunenvertreter, Behördenleiter. Auch sie werden durch die Arbeit der Migrantinnen vor lästigen Forderungen und Ansprüchen verschont. Dazu noch einmal dasselbe Gedankenexperiment: Gäbe es die Migrantinnen nicht, was wäre dann? Dann wären insbesondere die Mängel, die Lücken, die unzumutbaren Zustände in der Altenversorgung noch viel eklatanter und sichtbarer, vor dem Zorn der Bürger gäbe es kein Entkommen. Eine höchst brisante Situation, denn bezahlbare Lösungen sind nirgendwo in Sicht. Das wissen die Verantwortlichen genau. Von daher erklärt sich die charakteristisch zwiespältige Praxis, die sich heute eingespielt hat: Offiziell wird die Hausarbeits- und Pflegemigration ,illegal“" genannt, damit verurteilt, ja kriminalisiert. Inoffiziell jedoch wird sie stillschweigend und massenhaft toleriert. Eine bewusste und systematische Duldung also, längst als normal akzeptiert. Die ,große Scheinheiligkeit“ (zitiert nach Metz in: Das Parlament, 15.1.07) - so hat das Klaus J. Bade genannt.

Auch hier also ein Pakt des Wegschauens, ein Stillhalteabkommen in diesem Fall zwischen Politikern und betroffenen Familien. Dies wird möglich, weil die Migrantinnen die drängendsten Versorgungslücken überbrücken. Sie sind unsichtbar, die stillen Helferinnen im Innern. Und sie sind unverzichtbar, wesentliche Stützen für den Alltagsablauf in modernen westlichen Gesellschaften. 


\section{Versorgungslücken und globale Betreuungsketten: wie sich in der Heimat der Migrantinnen die Familien verändern}

Viele der Frauen, die im Ausland arbeiten, haben zuhause selbst Familie. Sie haben Mann und Kinder in der Heimat zurücklassen müssen, weil es dort kaum Verdienstmöglichkeiten gibt. Dabei sind es vielfach gerade die Kinder, die den Anstoß zur Migration geben. Die Frauen wollen Geld verdienen, um den Kindern eine bessere Zukunft bieten zu können. Dafür nehmen sie lange Zeiten der Trennung in Kauf und das Leben in der Fremde mit seinen Belastungen. Galt früher als Liebesbeweis, dass man zusammenbleibt, was immer auch kommt, so finden wir in der globalisierten Welt zunehmend das Gegenteil als Gebot: Wer seine Familie liebt, der verlässt sie, um anderswo die Grundlagen für eine bessere Zukunft zu schaffen. In einem Roman von Michelle Spring wird dies prägnant zusammengefasst: „Für Hausarbeitsmigrantinnen rund um den Globus bedeutet Liebe vor allem eines - Liebe bedeutet WeggehenMüssen“" (Spring 1998: 63).

Die Frage ist freilich, wie die Kinder während der Monate oder Jahre der Trennung versorgt werden. Nach vorliegenden Untersuchungen geschieht dies meist über neu entstehende Formen der Arbeitsteilung zwischen den Frauen. In der Regel setzen die Migrantinnen andere Frauen ein, die an ihrem Heimatort leben (z.B. Großmütter, Schwägerinnen, Nachbarinnen). Indem sie diese mit Geld und sonstigen Geschenken unterstützen, versuchen sie, Betreuungsdienste für die eigenen Kinder zu sichern. In der Folge entstehen transnationale Formen der Mutterschaft und globale Betreuungsketten, die sich über Länder und Kontinente spannen. Das sieht etwa so aus: In einer Familie der Zweiten bzw. Dritten Welt übernimmt die älteste Tochter die Betreuung ihrer jüngeren Geschwister; ihre Mutter wird dadurch freigestellt und kann vor Ort die Kinder einer anderen Frau betreuen, wodurch sie ein wenig Geld verdient; die andere Frau ist in eines der Wohlstandsländer des Westens gegangen und dort als Kindermädchen tätig. Solche grenzüberschreitenden Betreuungsketten finden wir z.B. in den Wanderungsbewegungen zwischen West- und Osteuropa: Da gehen Frauen aus Polen nach Deutschland, um in Mittelschicht-Familien auf die Kinder aufzupassen; und währenddessen kommen Frauen aus der Ukraine nach Polen, um den Haushalt und die Kinder der polnischen Arbeitsmigrantinnen zu versorgen. 


\section{Globale Versorgungshierarchie}

Die amerikanische Soziologin Arlie Hochschild hat den Verlaufsplan solcher Bewegungen in einem Satz zusammengefasst: „Motherhood is passed down the hierarchy of nation, ethnicity, race" (Hochschild 2000). Oder allgemeiner gesagt, im globalen Zeitalter entsteht eine Delegationshierarchie, eine neue globale Hierarchie. Die Versorgungsarbeit im Privaten wird auf der Stufenleiter der Nationen, Hautfarben, Ethnien von oben nach unten weitergegeben. Dabei schwinden von Stufe zu Stufe die Chancen für angemessene Versorgung und menschenwürdige Pflege. Bis irgendwann, auf der untersten Stufe, gar nichts mehr bleibt. Wenn Polinnen nach Deutschland fahren, um die familialen Alltagsarbeiten in deutschen Familien zu erledigen; und wenn Ukrainerinnen nach Polen fahren, um die Alltagsarbeiten in polnischen Familien zu erledigen; wer übernimmt dann die familialen Alltagsarbeiten in der Ukraine? Wer sorgt für die Kinder der ukrainischen Frauen, wer betreut ihre Eltern?

Empirische Untersuchungen zeigen durchgängig: Für die, die sich am je unteren Ende der Hierarchie befinden, sind die Folgekosten erheblich. Zum Beispiel die in der Heimat zurückgebliebenen Kinder: Die beauftragten Großmütter, Tanten, älteren Schwestern sind nicht selten überfordert, haben nicht genug Zeit oder Kraft, oder sind auch zu alt oder zu krank, um mit den zusätzlichen Anforderungen fertig zu werden. Dann sind die Kinder mehr bis minder sich selbst überlassen; werden vernachlässigt; werden zwischen verschiedenen Haushalten hin- und hergeschoben. Die Väter sind in dieser Situation kaum eine Hilfe: Nicht wenige haben schon vor Jahren die Familie verlassen und die Verantwortung für ihre Kinder ganz den Müttern zugeschoben; andere sind zwar geblieben, kommen aber mit der neuen Situation nicht zurecht, mit der Tatsache, dass die Frau im Ausland arbeitet und dass sie es ist, die die Familie ernährt; sie sind in ihrem Selbstverständnis verunsichert und nicht fähig oder nicht willens, für die Kinder zu sorgen. Die Folge ist, dass diese oft auch emotional vernachlässigt werden und leiden. Sie fühlen sich alleine gelassen und einsam, sie sehnen sich nach der unerreichbaren Mutter.

\section{Die Ängste der Mütter}

Auch für die Mütter ist die Situation alles andere als einfach. Einschlägige Untersuchungen zeigen, dass die Frauen selbst enorm unter der Trennung leiden. Sie haben Sehnsucht nach den Kindern; sie sind traurig, weil sie deren Aufwachsen nicht miterleben; sie sind von der ständigen Sorge belastet, ob die beauftragten Großmütter, Tanten, Nachbarin- 
nen das Kind gut versorgen; und sie haben gleichzeitig Angst, dass die Kinder sich ihnen entfremden und die Mutter vergessen.

So gesehen ist die Geschichte der Hausarbeitsmigrantinnen keine Geschichte des Gewinns, sondern vor allem der Verluste. Bekannt ist das Stichwort vom ,brain drain“, das auf die Abwanderung der Hochqualifizierten verweist und die Probleme, die sich für die betroffenen Gesellschaften daraus ergeben. Weit weniger bekannt ist, dass es auch so etwas wie einen „care drain“ gibt, also die Abwanderung derer, die für die familiale Alltagsarbeit zuständig sind, und in der Folge eklatante Versorgungslücken.

\section{Globale Hierarchie statt globaler Gerechtigkeit}

Man kann, wie gesagt, die beschriebene Geschichte positiv deuten, als Wohltat für alle. Eine solche Sichtweise ist angenehm, weil sie uns - die Bewohner und Bewohnerinnen der Ersten Welt - von unbequemen Fragen befreit. Das Problem ist nur, dass die harmonische Perspektive lückenhaft ist: Sie vergisst, dass Kosten und Nutzen asymmetrisch verteilt sind. Der Einsatz von Hausarbeitsmigrantinnen erzeugt neue Formen der sozialen Ungleichheit, das Wohlstandsgefälle zwischen armen und reichen Nationen reicht bis in die Küchen und Kinderzimmer hinein.

Gleichzeitig ist angesichts der beschriebenen Bedingungen zu erwarten, dass sich in Zukunft immer mehr Formen solcher privaten internationalen Vernetzung herausbilden werden. Wo die Grenzen zwischen Ost und West fallen, wo arme und reiche Nationen näher zusammenrücken (und selbst eine restriktive Abschottungspolitik wird dies auf Dauer kaum ändern), da werden die Wohlstandsländer des Westens weiter starke Anziehungskraft haben. Solange in diesen Ländern gleichzeitig die öffentliche Infrastruktur fehlt, die den einheimischen Frauen eine gleichberechtigte Berufsteilhabe erlaubt, solange werden diese Frauen weiter nach privaten Nischen, Notlösungen, Überlebensstrategien suchen.

In dieser Konstellation ist die internationale Arbeitsteilung immer weniger ein abstraktes Ereignis, das irgendwo zwischen Erster und Dritter Welt angesiedelt ist. Nicht nur in Industrie und Gewerbe, auch in der Familie werden zunehmend Arbeitsmigranten beschäftigt, genauer: Arbeitsmigrantinnen, Frauen ausländischer Nationalität. Im Zeitalter der politischen Umbrüche und Wanderungsbewegungen vollzieht sich die internationale Arbeitsteilung immer mehr vor unserer Haustür, ja zum Teil direkt in unseren Familien und Wohnungen. 
Für Altkanzler Schröder waren die Angelegenheiten des familialen Alltags „Gedöns“, ein Bagatell-Thema also, weit weg von den großen Fragen der Welt. Aber schon die Frauenbewegung der 1970er Jahre wusste es besser. Einer ihrer zentralen Leitsätze hieß „The Personal is Political“" (Hanish 1970; der Term entspringt dem gleichnamigen Essay von Carol Hanish aus dem Jahre 1969), mit anderen Worten: die privaten Lebensformen sind nicht nur ein privates, sondern auch ein politisches Faktum. Sie sind ein zentraler Baustein im Gefüge der Gesellschaft, und nicht zuletzt im Gefüge der Ungleichheit im Geschlechterverhältnis. Heute, im Zeitalter der weltweiten Wanderungsbewegungen, können wir hinzufügen: „The Personal is Global“ (Hochschild 2003: 30). Das bedeutet frei übersetzt: Die Verwandlung der familialen Alltagsarbeit und die Entstehung einer transnationalen privaten Schattenwirtschaft in den Grauzonen der Legalität - dies ist nicht nur eine Sache des persönlichen Lebensstils oder des persönlichen Geldbeutels, sondern direkt verknüpft mit Fragen der globalen Gerechtigkeit und der globalen Ressourcenverteilung. „Und für Opa sorgt 'ne Frau aus Osteuropa“: diese Entwicklung ist nicht denkbar ohne die politische, ökonomische, soziale Hierarchie zwischen den Nationen.

\section{Literatur}

Bade, Klaus J. (2000): „Fleißig - billig - illegal”: Der Migrationsexperte Klaus J. Bade über die wirtschaftliche Bedeutung illegaler Einwanderer. Interview von Andrea Böhm mit Klaus J. Bade, in: Die Zeit, Nr. 27 vom 29.6.00.

Hanish, Carol (1969): The Personal is Political, in: Shulamith Firestone/ Anne Koedt (Hg.) (1970): Notes From the Second Year: Women's Liberation, New York.

Hochschild, Arlie/Anne Machung (1990): Der 48-Studen-Tag: Wege aus dem Dilemma berufstätiger Eltern, Wien.

Hochschild, Arlie Russell (2000): Global Care Chains and Emotional Surplus Value, in: Will Hutton/Anthony Giddens (Hg.): On the Edge. Living with Global Capitalism, London, S. 130-146.

Hochschild, Arlie Russell (2003): Love and Gold, in: dies./Barbara Ehrenreich (Hg.): Global Woman. Nannies, Maids and Sex Workers in the New Economy, London.

Metz, Johanna (2007): Illegale Einwanderer in Deutschland. Die große Scheinheiligkeit, in: Das Parlament, 15.1.07.

Rerrich, Maria S. (1993): Gemeinsame Lebensführung. Wie Berufstätige einen Alltag mit ihren Familien herstellen, in: dies./Karin Jurczyk 
(Hg.): Die Arbeit des Alltags: Beiträge einer Soziologie der alltäglichen Lebensführung, Freiburg im Breisgau.

Spring, Michelle (1998): Running for Selter, London. 
